\title{
LOS FANTÔMES, SPECTRES Y ESPRITS EN LOS ESCRITOS DE CALMET*
}

\section{The Ghosts, Spectres and Spirits in Calmet Writings}

\author{
Carme AGUSTÍ APARISI \\ Universidad Católica de Valencia San Vicente Mártir \\ carme.agusti@ucv.es
}

Fecha de envío: 01/07/2017

Fecha de aceptación definitiva: 13/04/2018

RESUMEN: El fantasma representa dentro de la literatura fantástica una transgresión de la realidad, es un ente sobrenatural anclado en un mundo pragmático, real. Pretendemos aunar, en este artículo, tradición antropológica y literatura, pues el motivo del fantasma se encuentra presente desde la antigüedad en el folclore de la mayoría de culturas. El concepto cristiano de la muerte y de la vida eterna otorgada por el arrepentimiento de los pecados se verá alterado cuando queden excluidos suicidas, asesinados o excomulgados. Estas almas en pena aparecerán en los relatos recopilados en el tratado de Calmet, que presentará de manera analítica, como crónicas o anecdotarios, historias de Fantômes, Spectres y Esprits, tópicos de la narratología de los cuentos de fantasmas anglosajones del siglo XIX que compartirán una misma estructura temática que las historias contadas por Calmet.

Palabras clave: fantasma; espectro; alma en pena; folclore; tradición.

ABSTRACT: In fantasy literature, the ghost represents a transgression of reality; it is a supernatural entity anchored in a pragmatic, real world. As the phantom motif

* Este trabajo se ha realizado en el marco del Proyecto del Plan Nacional de I+D+i del Ministerio de Economía y Competitividad FFI2015-64050: Magia, épica e historiografía hispánicas: relaciones literarias y nomológicas, dirigido por el Dr. Alberto Montaner. Del mismo modo, forma parte de las actividades del grupo de investigación "Humanidades Digitales» de la Facultad de Magisterio y Ciencias de la Educación de la Universidad Católica de Valencia San Vicente Mártir. 
has been present since antiquity in the folklore of most cultures, in this article, we intend to combine anthropological tradition and literature. The Christian concept of death and eternal life, granted by the repentance of sins, shifts when exclusions are made of suicides, the murdered and the excommunicated. These tortured souls appear in the stories compiled in Calmet's treatise, which presents analytically, as chronicles or anecdotes, stories of Fantômes, Spectres et Esprits in a narratology of $19^{\text {th }}$ century Anglo-Saxon ghost tales that share the same thematic structure as the stories told by Calmet.

Key words: ghost; spectrum; banshee; folklore; tradition.

\section{INTRODUCCIÓN}

Calmet escribe en pleno siglo XVIII uno de los tratados más relevantes de su época. Gran conocedor y estudioso de las Sagradas Escrituras y de la Biblia, su afán de alabar y magnificar la presencia y la ley de Dios en toda la creación le lleva a estudiar temas filosófico-teológicos, que han servido, posteriormente, como fuente de inspiración de escritores que contribuyeron en la creación literaria de relatos que, en muchos de los casos, bebían y se influenciaban de los escritos de este benedictino que escribió, casi exclusivamente, para magnificar a Dios y contribuir a su grandeza.

Su Traité sur les Apparitions des Esprits, et sur les Vampires, ou les Revenans de Hongrie, de Moravie, $\varepsilon c$. (1746) fue famoso y difundido por toda Europa. En plena plaga de vampirismo europeo, donde las exhumaciones de cadáveres en el centro de Europa reportaron atrocidades inimaginables, consentidas, la mayoría de las veces, por las mismas autoridades, el Tratado representó un compendio de todo el saber de la época, y de todos los casos de vampirismo recogidos por el abad en multitud de ejemplos ${ }^{1}$. La finalidad del benedictino era demostrar su tesis principal según la cual «Je pose d'abord pour principe indubitable, que la Résurrection d'un mort vraiment mor test l'effet de la seule puissance de Dieu» (Calmet, 1751: 4 [tome II]). Pero sus elucubraciones revirtieron, en la imaginación popular, alimentada por la superstición y la ignorancia, y posibilitaron que se reafirmaran como ciertas muchas de las historias que Calmet había recopilado y contado de otros famosos tratados que él había leído ${ }^{2}$, diarios ${ }^{3}$, incluso relatos de prestigiosos personajes y autores ${ }^{4}$.

1. Para ampliar esta información, consultar Agustí (2016).

2. La Gesta regum anglorum, Discours et histoires des spectres, visions et apparitions des esprits, anges, démons et ames, Campus Elysius Jucund, De masticatione mortuorum in tumulis, Magia posthuma, relation d'un voyage du Levant..

3. Le Mercure Galant y Le Glaneur de Hollande.

4. Flegonte, Tertuliano, Plutarco, Schertz, Harenberg, Bartholin, Malmesbury, Newbridge, Alciato, Trithemius, Bremensis, Tournefort, Le Loyer, Vinflow, Le Bruyn, Gaspar Reyes, Rehrius, Ranft... 
Nuestras anteriores investigaciones han versado en demostrar que muchas de las historias del abad sobre los revenants aparecen en los motivos folclóricos de Thompson (1955-58)5 y que provienen de la tradición oral y del folclore, especialmente de los pueblos del centro de Europa (Eslovaquia, Silesia, Moravia...), así como de las provincias griegas. Calmet es un gran recopilador, sus historias son cuentos que pueden ser tomados como materia literaria y que influenciarán a la literatura gótica posterior.

En nuestros anteriores estudios hemos abordado el segundo tomo del Traité de Calmet, es decir, investigamos sobre las causas del vampirismo y de las creencias en vampiros (revenants) en el centro de Europa. Pero Calmet comenzó su tratado con un primer volumen en el que literalmente disertaba sobre ángeles, demonios y almas retornadas de la muerte, titulado Traité sur les Apparitions des Anges $\&$ des Démons, et des Ames des Défunts.

Pretendemos, pues, en este artículo, en primer lugar aproximarnos a conceptos básicos elaborados por nuestro autor respecto a los Fantômes, Spectres y Esprits, términos que muchas veces ya habían sido formulados por autores anteriores a Calmet, o que también servirán de punto de partida para otros escritores posteriores a nuestro abad. Ideas que irán elaborando relatos antropológicos, pero también literarios, y que crearán muchos de los tópicos de una literatura "fantástica", en este caso sobre fantasmas, que tendrán su máxima expresión en la literatura gótica anglosajona. No es nuestro propósito analizar ni todos los relatos del abad ni cuentos de fantasmas de la literatura universal. Nos proponemos, por tanto, una vez hecho el desglose de conceptos, profundizar en algunos relatos de Calmet para describir y constatar que muchos de los tópicos de los cuentos de fantasmas que se reproducirán en relatos posteriores ya aparecían en las descripciones y explicaciones que recogía el benedictino en sus escritos. Partiremos de ejemplos concretos para comentar cómo el motivo pasará a la literatura con la creación de cuentos de fantasmas, sobre todo, en la literatura anglosajona, teniendo en cuenta que la visión literaria es panorámica y que no entraremos a analizar o comparar un corpus de cuentos de fantasmas. Por otra parte, también apuntaremos como idea novedosa para investigaciones futuras que los relatos

5. Hemos considerado también interesante rastrear en Motif-index of folk-literature todos estos tópicos. E: THE DEAD. E200-E599. GHOSTS AND OTHER REVENANTS: E251.2. Vampire brought to life. E251.3.3. Vampire sucks blood. E279.2. Ghost disturbs sleeping person. E422.1.9. Living corpse returns every night. E431.7. Beheaded man's head laid at feet to prevent return. E431.7.2. Decapitating in order to prevent return. E431.13. Corpse burned to prevent return. E439.5. Revenant forced away by fire. E541. Revenants eat. E541.4. Revenants drink. En muchos de los relatos que aparecen en el Traité, la línea que separa el término vampiro del término espectro o fantasma es muchas veces confusa. Indistintamente, los términos sirven para designar al chupador de sangre; sí que es cierto que, mayoritariamente, el abad se refiere a los vampiros como revenans. Lo mismo pasa con la clasificación de Thompson, indistintamente se utilizan dos términos para hablar de estas criaturas: vampire y ghost. 
del benedictino podrían ser un primer germen de «literatura fantástica» ${ }^{6}$, ya que podemos apreciar que características de esta literatura están presentes, sin lugar a dudas, en estas creaciones tratadísticas del siglo XVIII.

\section{APROXIMACIÓN A CONCEPTOS BÁSICOS: FANTÔMES, SPECTRES, ESPRITS}

Como intelectual de la época, nuestro autor está interesado por todas las cuestiones que se debaten en su tiempo, y así comienza su Traité sur les Apparitions, justificando la polémica que existe sobre este tema y afirmando que él pretende "traiter certe matiere pour voir à quel point de certitude on peut porter» (Calmet, 1751: 1 [tome I]), es decir, ¿hasta qué punto es cierto que tanto ángeles, como demonios y almas de hombres muertos, pueden aparecerse a los vivos?? Para ello, divide el tratado en cuatro grandes bloques:

1. Las apariciones de los buenos ángeles.

2. Las apariciones de los malos ángeles.

3. Las apariciones de las almas de los muertos.

4. Las apariciones de hombres vivos.

A partir de esta organización temática se centra en las apariciones de los buenos ángeles tanto en el Antiguo Testamento como en el Nuevo, se basa para ello en las opiniones de los judíos, los cristianos, los mahometanos y los orientales, así como se vale de lo dicho anteriormente por los egipcios, los griegos y los romanos. Pasa a constatar la presencia de los malos ángeles en las Sagradas Escrituras, y analiza cuestiones referentes a la magia, las brujas y la brujería; constata la presencia del demonio en las Sagradas Escrituras y su poder de posesión, así como sus posibles causas. Habla de los espíritus familiares de la antigüedad, y los clasifica en buenos y malos espíritus. Y pasa, finalmente, a relatar las Apariciones

6. Pensamos al igual que ROAs (2006) que durante el siglo XVIII las diversas formas de lo sobrenatural tenían una gran importancia en los relatos literarios representados por: la comedia de magia, el cuento maravilloso y la literatura de cordel. Añadiríamos, desde nuestro estudio, los relatos de Calmet, que como ya analizaremos están plenamente relacionados con textos literarios de fantasmas.

7. Es curioso constatar que, en pleno siglo XIX, se seguían interesando por las mismas cuestiones. El auge de la parapsicología, espíritus, médiums y sociedades espiritistas de la Inglaterra victoriana contribuyó a la proliferación de instituciones que pretendían investigar, desde la ciencia, fenómenos que en la época en la que escribe Calmet tenían una explicación sobrenatural. En 1885 Eleanor M. Sidgwick, conocida como una observadora extremadamente buena y una persona cuya formación matemática le otorgaba escepticismo y lógica, llevó a cabo una investigación psíquica sobre la cuestión del fantasma, "Sidwick surveyed four theories of phantasms of the dead: 1. the popular view of the actual existence in space of the apparitions; 2 . that the apparition has no actual existence in space but is a telepathic hallucination; 3. that the first appearance of the apparition is a subjective hallucinations and it is spread like a telepathic infection to other percipients; 4 . that there are subtle psychical influences in "haunted houses" which produce a hallucination in the percipient" (McCorristine, 2010: 175-176). 
de los Spectros: apariciones de almas probadas en las Escrituras; apariciones contadas por la Historia; sobre qué piensan los judíos, los griegos y los latinos sobre estos temas; de las apariciones de hombres vivos a otros hombres vivos; para finalizar su primer volumen argumentando las objeciones sobre estas apariciones y exponiendo sus conclusiones a esta primera parte del Tratado.

Su finalidad al escribir, él dice que es para formarse, para hacerse una idea «justa» de todo lo referente a las Apariciones de los Ángeles, de los Demonios y de las Almas separadas de los cuerpos, y afirma: "Mon but n'est point de fomenter la superstition» (Calmet, 1751: 2 [tome I]); sino que su intención consiste en investigar "quel point cette matiere étoit certaine ou inconue, vraie ou fausse, connue ou inconnue, claire ou obscure» (Calmet, 1751: 3 [tome I]). Como podemos apreciar su propósito es el de un investigador que a partir de unas tesis o hipótesis pretende demostrar qué hay de cierto en aquello que indaga, quiere: "montrer la vérité» (Calmet, 1751: 4 [tome I]) en bien de la religión y por su condición de cristiano. Reconoce que su primer gran objetivo «a été de parler des Vampires de Hongrie» (Calmet, 1751: 5 [tome I]), y plantea sus reflexiones desde una triple visión: «en Historien, en Philosophe, en Théologien» (Calmet, 1751: 7 [tome I]) para poder descubrir la verdad.

Escribe para "corriger les abus qui naissent de cette créance peu éclairée» (Calmet, 1751: 8 [tome I]). Según el abad, las Escrituras hablan de las Apariciones de Ángeles y de Almas separadas de su cuerpo, pero afirma que la manera en que Dios permite esas resurrecciones o apariciones «est cachée dans ses secrets» (Calmet, 1751: 10 [tome I]). Al referir las historias, que trabajaremos en este artículo, él mismo las califica de «fictions poëtiques» (Calmet, 1751: 14 [tome I]), relatos "fausses, ou comme très-douteuses, ou même comme fabuleuses" (Calmet, 1751: 14 [tome I]); y deja bien claro que estos no pueden perjudicar a los dogmas de la Religión y a las Apariciones que se describen como verdaderas en las Sagradas Escrituras. Y para concluir su introducción afirma: "Je déclare que je tiens pour vraies toutes les Apparitions rappottées dans les Livres sacrés de l'Ancien \& du Nouveuau Testament» (Calmet, 1751: 20 [tome I]).

El abad diferencia en primer lugar entre vampiros: "Revenans en Corps»y espíritus: "Revenans immatériels»; y los vampiros son: "Les Revenans de "Hongrie", ou les Vampires, [...] sont des hommes morts depuis un tems considérable [...] qui sorten de leurs tombeaux \& vienent inquiéter les vivans, leur sucent le sang, leur apparoissent, [...] enfin leur causent souvent la mort» (Calmet, 1751: 1 [tome II]). Pero Calmet, en muchas de sus historias, emplea indistintamente en su volumen II el término Revenans, Fantôme, incluso Spectre. Si el fantasma es la imagen de una persona muerta que se aparece a los vivos y el espectro es una aparición aterradora, también de un muerto; es factible que ambos términos funcionen como sinónimos, teniendo en cuenta, a su vez, que el Revenant es el muerto que regresa de la tumba para alimentarse de los vivos, concepto que, por la succión de la sangre, no participa exactamente de las características de los dos anteriores. En 
cambio, el espíritu (Esprit) se refiere a un ser inmaterial y dotado de razón, alma racional, parte incorporal del ser humano por oposición a su cuerpo, a la materia.

La teoría sobre los "espíritus» de Calmet es muy interesante. Según nos dice, los ángeles y los espíritus de los muertos pueden aparecerse a los vivos, siempre con autorización de Dios, porque no son del todo inmateriales, ya que, si así fuera, sería muy difícil explicar cómo son posibles estas apariciones, y estas son, exclusivamente, espirituales. Según él, el alma posee una cierta cantidad de materia que hace posible que pueda moverse a su voluntad, pueda condensarse y transformarse en aire visible a nuestros ojos. Ya que respecto a la forma de aparecerse de los buenos ángeles afirma: "La maniere la plus ordinaire dont les bons anges apparoissent dans l'Ancien \& dan le Nouveau testament, est sous la forme humaine" (Calmet, 1751: 12 [tome I]). Por tanto, deja claro que los buenos ángeles sí que se aparecen bajo apariencia humana ${ }^{8}$, pero siempre por expresa voluntad de Dios: "De tout ce que nous venons de dire il résulte, que les Apparitions des bons Anges sont non-seulement possibles, mais aussi très-réelles» (Calmet, 1751: 35 [tome I]); es decir: "Ni l'Ange, ni le Démon n'ont pas plus de privilége à cet égard sur la matiere, que l'ame de l'homme sur son prope corps. Ils ne peuvent ni modifier la matiere, ni la changer, ni lui imprimer des actions \& des mouvemens que par le pouvoir de Dieu»(Calmet, 1751: 434 [tome I]).

Calmet diferencia entre: 1. Buenos Ángeles: que anuncian buenas nuevas, y si son males futuros, ayudan a los hombres a prevenirlos. 2. Malos Ángeles: que predicen maldades, guerras, calamidades públicas y son efectos de la cólera de Dios. 3. Las almas de los difuntos: que regresan para cumplir fines cristianos, decir misas, hacer peregrinaciones, dar limosnas...; o avisar a los vivos y prevenirles para que se arrepientan de sus pecados.

Y afirma:

1. Que los ángeles, demonios y almas separadas del cuerpo son puros espíritus separados de toda materia.

2. Que las sustancias espirituales solamente pueden aparecerse a los hombres por orden de Dios.

3. Que para poder aparecerse, hablar, caminar, comer... pueden condensar aire o substituir otros cuerpos terrestres y sólidos capaces de poder proporcionarles la visibilidad.

4. Que no podemos conocer el porqué de estas substancias sólidas y que pensamos que las substancias espirituales tienen ese poder de producir fuerzas que dependen, exclusivamente, de Dios.

5. Que hay apariciones que pueden predecir hechos futuros para oponerse al demonio (Calmet, 1751: 436 [tome I]).

8. Según Aguirre (2014: 55), las tres apariencias más comunes de los fantasmas en la antigüedad eran: negro, blanco o como humo. También GonZÁlez-Rivas (2014: 273) apoya esta tesis: negro, blanco o etéreo. 
Respecto a los espíritus malvados dice:

Les mauvais esprits apparoissent aussi quelquefois sous la figure d'un lion, ou d'un chien, ou d'un chat, ou de quelqu'autre animal, comme d'un taureau, d'un cheval, ou d'un corbeau: car les prétendus Sorciers \& Sorcieres racontent, qu'au Sabbat on le voit de plusieurs formes différentes, d'hommes, d'animaux, d'oiseaux, soit qu'il prenne la forme de ces animaux, ou qu'il se serve des animaux mêmes comme d'instrumens pour tromper ou pour nuire (Calmet, 1751: 44 [tome I]).

Nuestro autor estuvo influenciado en sus escritos por Pierre Le Loyer ${ }^{9}$ que ya por el año 1605 hablaba de "visions d'Esprits, qu'on appelle Spectres» (Le Loyer, 1605: 1), a los cuales definía así: "Spectre est une imagination d'une substance sans corps, qui se presente sensiblement aux hommes contre l'ordre de nature, \& leur donne frayeur» (Le Loyer, 1605: 2). Igualmente, el abad influyó en gran cantidad de autores posteriores, uno de los cuales fue Collin de Plancy que en su Histoire des Vampires et des Spectres Malfaisans (1820) define el término Vampires como: "Ces morts qui sortent en corps et en âme de leurs cercueil pour venir sucer les personnes vivantes, leur donner la mort, et s'engraisser de leur sang» (Collin de Plancy, 1820: 2) ${ }^{10}$. Respecto a otros conceptos referentes a esta investigación, dice Collin en su obra Dictionnaire Infernal (1844) que Fântome son: "Esprits ou revenants de mauvais augure qui effrayaient fort nos péres» (1844: 208), y termina remitiendo a términos como Apparitions, Esprits, Revenans, Spectres..., lo cual viene a constatar que todos ellos pertenecen a un mismo ámbito.

Veamos pues cómo define a los Esprits: «Les anciens ont cru que les esprits, qu'ils appelaient démons ou génies, étaint des demi-dieux. [...] Il y a [...] des philosophes qui se sont imaginé que ces esprits n'étaient que les âmes des morts qui, étant une fois séparées de leurs corps, erraient incessamment sur la terre» (Collin de Plancy, 1844: 201). Para los Spectres, afirma que son: «Sorte de substance sans corps, qui se présente sensiblement aux hommes, contra l'ordre de la nature» (Collin de Plancy, 1844: 455).

Tal y como refiere Lecouteux (2009b: 7-8), la lengua francesa dispone de numerosos términos para designar "ces morts inquiétants". "Fantôme» que evoca la idea de ilusión y fantasmagoría. "Spectre» sobre el que se cierne un concepto de horror y de miedo. "Esprit", manifestaciones inexplicables que llevan a la perplejidad al ser humano y, por último, «Revenant», que nos sugiere el retorno de un muerto.

9. LE LOYER (1605) trata, en su extensa obra compuesta de ocho libros, muchas de las reflexiones que después recogerá Calmet: definition de Spectres, des Sorciers, de le Diable, de apparut, histoires de Spectres, des superstitieux, des Esprits malins... Es interesante su Premier libre, Chapitre Premier: De la difference des Spectres, Visions \& Fantomes; des Anges, Démons \& Ames; sobre todo para entender la influencia que ejercerá sobre los escritos del abad y porque escribirá un siglo antes que él.

10. Consultando la obra de Collin de Plancy, podemos apreciar en la página 3 que toma literalmente la definición de Calmet y así lo remarca. 
Los términos, como podemos observar, presentan matizaciones tan sutiles que, en muchas ocasiones, llegan a superponerse. Roas (1999: 93) nos dice al respecto: "El fantasma es un muerto incorpóreo», concepto que nos provoca terror debido a la posibilidad de la animación de un muerto que regresa a la vida. Morillas (1999: 269), igualmente, nos habla del fantasma como «espectro condenado", una amenaza sobrenatural que "no viene, al parecer, a otra cosa que a dañarnos" (p. 270) que nos provoca horror y estremecimiento. Y, por último, González Salvador (1999: 295) nos refiere que son palabras del mismo contexto: «visión, espectro, sombra, aparición, espíritu, alma y aliento»; aunque matiza que «el fantasma suele ser, preferentemente, un muerto o, mejor dicho, "el (muerto)" que vuelve».

Pero, ¿cuáles son las causas que hacen que puedan aparecerse estos espíritus y qué clases de espíritus se aparecen? Para Calmet, los espectros o almas de los muertos que molestan a los vivos son de dos clases: 1. Las almas del Purgatorio. 2. Las almas de aquellos que han sido ejecutados y piden venganza, así como la sepultura para sus cadáveres. Habla, a su vez, de dos tipos de Espectros: «1. Les uns qui apparoissent aux hommes pour leur nuire, our pour les tromper, our pour leur annoncer des choses futures. 2. Les autres Spectres [...] des maisons» (Calmet, 1751: 285 [tome I]). Evidentemente, en nuestro estudio investigamos a los primeros.

Existe una clara relación entre el concepto de la "buena muerte» y el de la "mala muerte» medieval ${ }^{11}$, y la creencia en la aparición de estos espíritus o espectros. Ya desde la antigüedad, el respeto por los ritos funerarios era imprescindible para conseguir el descanso eterno. Calmet confirma la teoría de la buena muerte griega: «Ils croyoient aussi que les ames n'étoient point en repos, \& qu'elles rodoient autour de leurs cadavres tandis que leurs corps n'étoient pas inhumés» (Calmet, 1751: 31 [tome I]). La buena muerte y el entierro suponen una gran preocupación para los pueblos antiguos. Y afirma Calmet: "Les Rabins écrivent, que l'ame n'est reçûe dans le Ciel qu'après que le corps grossier est enterré \& entierement consumé» (Calmet, 1751: 377 [tome I]). En la Grecia antigua, para evitar que los muertos regresaran, se incineraban sus cuerpos, ya que se pensaba que su extinción total confinaba su espíritu en el Hades y lo alejaba del posible retorno con los vivos, el fracaso de estos ritos funerarios era el causante del regreso de los muertos a la vida (Roas, 1999: 94). Si la transición se realizaba bien, el muerto podía alcanzar el «lugar correcto» para su reposo que era la tumba para su cuerpo

11. Los ritos funerarios se basaban en "Les rites de passage», que eran tres: el rito de separación (lavar el cuerpo y llevarlo al cementerio), el rito del margen (velar el cuerpo del difunto) y el rito de agregación (la comida funeraria en honor al cadáver). Si uno de estos tres ritos se incumplía, hablaríamos de la "mala muerte», y los muertos serían un peligro para todos, ya que tendrían la posibilidad del retorno. Por tanto, no pueden pasar al más allá quedándose bloqueados. Hablaríamos, pues, de "morts purs et morts impurs". Los puros (cistyj) que pasaban directamente al mundo de sus ancestros; y los impuros (necistyj) que transgredían, constantemente, la frontera entre este mundo y el más allá (Lecouteux, 2009a: 33-38). 
y el Más Allá para su espíritu. Pero, si esta transición no era bien realizada, se producían los diversos motivos para la vuelta de los muertos.

En la Roma antigua, los ritos funerarios eran imprescindibles para poder dar reposo eterno y descanso a los muertos. Se creía que «les Mal morts» se debían a causas como: las muertes violentas (asesinatos, suicidios); los soldados que morían en determinadas circunstancias y no recibían los ritos adecuados; las muertes prematuras; o aquellas que quedaban insepultas por diversas razones ${ }^{12}$, lo cual posibilitaba una tropa de revinientes y de fantasmas, de espíritus que no podían encontrar su reposo eterno y la paz. El arte del bien morir reportaba la paz a los muertos. Según Lecouteux será en el siglo XII, cuando se produzca la interpretación cristiana de los revenants, a partir de las reinterpretaciones de Tertuliano y san Agustín, que propiciarán la siguiente explicación: "Dieu autorise parfois les anges déchus à se glisser dans les cadavres et à les animer» (2009: 59). Dejando muy claro que «le revenant est une sorte de démon, qu'il perd sa corporéité et devient un fantôme, une image, un simulacre de corps, qu'il se transforme en âme en peine ou en damné» (Lecouteux, 2009b: 60).

Entre las razones de la vuelta post mórtem encontramos: la venganza, atormentando en forma de espíritu; ya sea porque no se le guardó el debido respeto, murió antes de acometer un encargo, se ha de proteger a alguien... (Roas, 1999: 94), el anuncio de una muerte, de una enfermedad o la búsqueda de consejo. Pero también podemos encontrar otros motivos relacionados directamente con el folclore y la tradición, como pueden ser: infligir un castigo, cumplir un compromiso, porque no pueden reposar..., según Thompson: E230-E247 Return to Inflict Punishement. E340-E353 Return from Dead to Replay Obligation. E410-E419 The Unquiet Grave; y así, tal y como nos dice Ezama (1999: 328): "La desaparición del fantasma se produce en muchos casos cuando cesa o se satisface la causa de su aparición, [...] pero también por otras razones, la incineración del cuerpo o las lágrimas de arrepentimiento».

La idea neoplatónica de la vida después de la muerte asentó la creencia en espectros y fantasmas, así como en todo tipo de revenants, finalmente con el cristianismo se completó la idea de que el alma continuaba su vida en el Más Allá después de la muerte, esperando a la resurrección de los muertos. El concepto de redención posibilitó que las almas de los pecadores pudieran redimirse, a cambio de recibir los últimos sacramentos y de arrepentirse de los pecados. Pero eran excluidos de estos beneficios «tous ceux qui n'ont pas reçu l'extrême-onction ou qui n’ont pas été inhumés en terre consacrée» (Marigny, 2010: 22). Es decir, suicidas,

12. Delgado (2014: 29) habla de espíritus difíciles de neutralizar: fallecidos por causas violentas, cremaciones incorrectas, ahogamiento o muertos devorados por animales. BuxTON (2014: 47-49) habla de: no enterrados (Ataphoi), muertos por violencia (Biaiothanatoi) y muertos insatisfechos (Aoroi). OGDEN (2014: 102) introduce también los bebés muertos y los niños, los condenados a muerte injustamente, los suicidas y los muertos por amor. 
excomulgados, asesinados... que son "almas en pena» (revenants) a la espera de su redención en el Purgatorio ${ }^{13}$.

Por tanto, podemos afirmar, para finalizar este apartado, que Calmet llegará a una serie de conclusiones respecto a su Traité sobre las Apariciones. Establecerá que todas aquellas apariciones que se producen en las Sagradas Escrituras son ciertas y obra de Dios, pero que también hay otras muchas apariciones que son «'ouvrage de la fourberie, de la malice des hommes, de la subtilité des charlatans, \& de la souplesse des joueurs des passe-passe» (Calmet, 1751: 468 [tome I]). Habla de imaginación, de simplicidad, de superstición, de excesiva credulidad, de la ignorancia que ha dado lugar a muchos relatos de pretendidas apariciones, ya que para poder explicar la materia de las Apariciones sería necesario: 1. Conocer perfectamente la naturaleza de los Espíritus, de los Ángeles y de los Demonios, y saber si las almas son puramente espirituales, o bien aún pueden tener alguna relación con cuerpos aéreos, sutiles, invisibles. 2. Si después de la muerte del alma, esta conserva poder sobre su propio cuerpo o sobre otros, por ejemplo, sobre el aire u otros elementos. 3. Si los Ángeles y los demonios tienen el mismo poder sobre los cuerpos para espesar el aire, para hacer aparecer fantasmas... 4. Si pueden hacer todas estas cosas, con o sin el permiso de Dios.

Y esto es lo que se propone demostrar con su tratado, pero son tantos los interrogantes que lanza Calmet que llega a aseverar que el lector pensará de él que arroja muy poca luz sobre esta materia, que duda, que no lo sabe cierto. Que solo acepta aquello que:

Ma Religion m'enseigne sur la nature des Ames, des Anges \& des Démons, je dirai qu'étant purement spirituels, il est impossible qu'ils apparoissent revêtus d'un corps, quel qu'il soit, à moins d'un miracle: supposé toutefois que Dieu ne les ait pas créés naturellement capables de ces opérations, avec subordination à sa volonté souverainement puissante, qui ne leur permet que rarement de mettre en éxecution cette faculté de se faire voir corporellement aux mortels (Calmet, 1751: 484 [tome I]).

Y, por tanto, nos contará una serie de ejemplos que son algunas de las historias que analizaremos en esta investigación.

13. El Purgatorio o purgatorius, que purifica, es el estado transitorio de purificación y expiación de los pecados, desde la perspectiva de la religión. Según la teología católica, las almas que mueren en pecado los purgan en este estado transitorio antes de poder acceder al Paraíso, hasta alcanzar el perdón y la redención. Fue Dante Alighieri (1265-1321), en su obra Divina Commedia, quien hizo una descripción, por primera vez, del Purgatorio, que dividió en siete giros, en los cuales se expiaban los siete pecados capitales. Es muy curioso que algunos de los cantos de la obra coincidan temáticamente con las causas de espectros y aparecidos que se producían por motivo de la "mala muerte»: Canto III Antepurgatorio: las almas de los excomulgados (p. 251). Canto V Antepurgatorio: Los negligentes muertos violentamente (p. 259). Canto VI Antepurgatorio: Los negligentes muertos violentamente (p. 263). Canto XXV Infusión del alma en el cuerpo, cuerpos aéreos después de la muerte (p. 350). 


\section{LOS CASOS DE CALMET Y SU RELACiÓN CON LA LITERATURA}

En los relatos ${ }^{14}$ seleccionados para este estudio aparecen espíritus benévolos y otros malévolos. Presentaremos, a continuación, las historias recogidas por Calmet, según los capítulos trabajados y la clase de espíritus que son. En el capítulo XXVII, encontramos dos historias de espectros benévolos; en primer lugar, el caso de un amigo que recurre a otro para remediar su excomunión y poder alcanzar el perdón y el reposo. En el segundo relato, se cuenta la historia de una mujer muerta que regresa para purgar su promesa de peregrinar y, así, poder alcanzar el reposo eterno.

En el siguiente capítulo, el XXXV, nos centraremos en tres historias en las que los espíritus malignos regresarán para vengarse. En el primer relato, el espíritu lo hará de su hijo; en el segundo, y como es un soldado, para vengarse de los romanos que lo mataron y que eran sus enemigos; y en el tercero, para predecir males futuros.

En el capítulo XXXVIII, de nuevo, serán los espíritus benignos, los protagonistas de seis relatos. Comenzaremos con la narración de dos amigos, que se prometen volver de la muerte para darse noticias del Más Allá. El segundo relato se centra en la aparición de un fantasma que vuelve para pedir misericordia por sus pecados. El tercero narra el pacto de dos amigos de regresar de la muerte, para darse noticias y la vuelta de uno de ellos para cumplir la promesa y dar esa información. Otra narración refiere la historia de dos monjes que prometen, también, regresar después de muertos, para darse fe de la existencia de la vida eterna y de la felicidad de los elegidos. La penúltima habla de un amigo que vuelve después de muerto para advertir de que si se quiere gozar de la vida eterna hay que llevar una existencia cristiana sin pecado. Por último, cerraremos este capítulo con la narración de un fantasma que clama venganza por su asesinato.

Para finalizar nuestro análisis, relataremos la historia del capítulo XXXIX, en la que un amigo ha retornado de la muerte para pedir que se cumpla un encargo, y hasta que este no se haya cumplido el fantasma no desaparecerá.

El capítulo XXVII se titula: Gens qui vont en pélerinage après leur mort ${ }^{15}$. En este capítulo, Calmet nos relata dos historias que muy bien podemos enmarcar como relatos de fantasmas (esprits), pues son muertos que vuelven a la vida

14. Las historias analizadas en este trabajo pertenecen todas al tomo II del Tratado de Calmet.

15. Calmet es un recopilador de historias, su Traité es un conjunto de relatos que le han contado, ha oído, ha leído en prensa de la época, le han relatado a través de cartas que ha recibido...; y su función como buen benedictino es presentar estas historias para refutar estas creencias en revenants y esprits. Su única tesis consiste en aseverar que solamente Dios tiene poder para resucitar a los muertos. Hemos profundizado en otros artículos, las características de sus relatos de revenants (AGUSTí, 2016), y así constatar que el autor siempre recurre a otros autores sobre los que basar sus relatos. En este caso que nos ocupa, Calmet menciona a Melchior L. y su obra De Statu Mortuorum. Es curioso constatar que este mismo relato es recogido, posteriormente, en otro tratado publicado en París, el año 1819: Réalité de la Magie, et des Apparitions, ou Contrepoison du Dictionnaire Infernal, que en el apartado 
para poder saldar situaciones no resueltas. El primero de estos relatos comienza diciéndonos: "Un ecolier de la ville de Saint-Pons près Narbonne étant décédé dans l'Excommunication, apparut à un de ses amis, \& le pria d'aller dans la ville de Rhodès demaner son absolution à l'Evêque» (Calmet, 1751: 113 [tome II]). La situación está clara, habiendo muerto en pecado, y, por tanto, siendo excomulgado, nuestro estudiante no recibirá el perdón eterno, a menos que obtenga la absolución del obispo de Rodas, por lo cual, recurre a su mejor amigo para que cumpla con el peregrinaje y pueda alcanzar su descanso eterno. Es remarcable, en esta historia, que este "espíritu» es benevolente ya que acompañará durante el viaje al protagonista y, además, le auxiliará y ayudará durante el trayecto: «'Esprit qui l'accompagnoit sans en être vû, lui montroit le chemin, \& ôtoit la neige» (Calmet, 1751: 113 [tome II]).

El segundo relato de este capítulo, parte de una carta recibida por Calmet fechada el 5 de abril de 1745, y que él nos cuenta de la siguiente forma: un hombre viudo de Létraye volvió a casarse y la primera esposa se apareció a la nueva "a onze heures du soir du jour de ses Nôces, sa femme apparut \& parla à la nouvelle Epouse» (Calmet, 1751: 114 [tome II]), obligándola a realizar 7 peregrinaciones en nombre de aquella. La muerta formó parte del peregrinaje y cuando ya se había realizado la mitad del mismo, la difunta desapareció. En este caso la mujer estaba ligada a una promesa que no había podido llegar a cumplir. "Elle étoit liée par le voeu ou la promesse qu'elle avoit faite d'accomplir ces pélerinages, dont elle chargea l'autre jeune femme qui lui succéda» (Calmet, 1751: 115 [tome II]). Estaríamos hablando, por tanto, de un «espíritu» que retorna para terminar una empresa piadosa no realizada, cuya alma purga, en esta vida, hasta poder realizar su peregrinaje.

La diversidad de las situaciones no resueltas es uno de los tópicos literarios más frecuentes de los relatos de fantasmas. Como hemos apreciado, en este capítulo, el retorno es en ambos casos piadoso, relacionado, exclusivamente, con el perdón para poder alcanzar la salvación eterna. Pero el motivo literario se diversificará, teniendo en cuenta que la base primigenia es la misma en ambos casos (tratados/literatura). Veamos, pues, las variaciones que nos presentan, de este tópico, algunos relatos literarios. Los cuentos de fantasmas que vuelven para saldar promesas no cumplidas, cuentas no pagadas o hechos no resueltos, predecir el futuro o la muerte de alguien cercano están muy presentes en la narrativa desde la antigüedad. Ya en la época de Plinio, y mediante la técnica narrativa de la carta, este comienza una disertación para constatar si existen los fantasmas en su Carta a Sura (Epistulae, VII, 27). El autor confirma y cree en ellos, y nos relata la historia que le sucedió a Curcio Rufo, al cual se le apareció una «figura de mujer, de altura sobrehumana y más hermosa de lo normal» que le anunció su futuro y su muerte,

de Apparitions, Fantomes, Revenans, punto 6. Apparition d'un jeune excommunié (p. 12) cuenta literalmente la misma historia recogida en Calmet. 
la cual se cumplió; una figura femenina que en el relato, evidentemente, se identifica con un fantasma. Otro relato de fantasmas de la literatura anglosajona es $A$ True Relation of the Apparition of One Mrs Veal (La aparición de Mrs. Veal) (1705) de Daniel Defoe, que se centra en la muerta que se aparece a una amiga bajo la forma de un fantasma, y que regresa para disculparse por la pérdida de su mutua amistad. Cuento donde la figura del espíritu que vuelve es para pedir perdón.

En su obra Infernaliana (1822) Nodier nos habla de "Spectres qui vont en pélerinage ${ }^{16}$, donde relata la historia de Pierre d'Engelbert y el espíritu de un soldado que Pierre había enviado como servidor al rey de Aragón. Muerto el joven, se le apareció a Pierre para expiar sus pecados por los males cometidos, y para que repartiera sus posesiones entre los pobres, se dijeran misas por su alma y, desde ese momento, el pobre Sancho, que así se llamaba el soldado, "ne revint plus» (Nodier, 1822: 79). Encontramos, también, Wandering Willie's Tale (Relato de Willie el Vagabundo) (1824) de Walter Scott, que, basándose en una leyenda escocesa, nos cuenta la historia de una deuda que se paga a un muerto y, al no tener pruebas de este pago, el deudor vuelve al más allá para recoger el recibo del pago y cancelar la deuda. Por último, comentaremos el relato At Chrighton Abbey (En la abadia de Chrighton) (1871) de Mary Elizabeth Braddon, historia sobre una terrible maldición que pesa sobre los primogénitos de una antigua familia. Edward, primogénito, promete que volverá a la hora del baile de la cacería en la que se encuentra. En este relato la promesa del regreso se manifiesta en forma de fantasma, aparición que presagia la muerte, que finalmente se cumplirá.

El segundo capítulo de nuestro estudio es el XXXV: Exemples de Fantômes qui ont apparû vivans, E ont donné plusieurs signes de vie. En este capítulo Calmet refiere ejemplos de fantasmas recogidos en el libro Des spectres de Le Loyer ${ }^{17}$, espíritus que, en este caso, han sido poseídos por un demonio para volver a la vida, siempre con el consentimiento de Dios. Veamos cómo lo relata Calmet: «Le Loyer, dans son libre des Spectres, soûtient que le Démon peut faire des mouvements extraordinaires \& involontaires aux possédés. Il peut donc aussi, avec la permission de Dieu, donner le mouvement à un homme mort \& insensible» (Calmet, 1751: 151 [tome II]).

Nos centraremos en tres historias, por lo curioso del caso: cabezas que hablan ${ }^{18}$ como espíritus vengativos, augurando males futuros. El primer caso es el de

16. Para el relato original, consultar bibliografía Infernaliana (pp. 76-79).

17. LE LOYER, en su libro Discours et Histoires des Spectres, Visions et Apparitions des Spectres, Anges, Demos (1605), y concretamente en el Livre Premier, Chapitre Premier, habla de los espectros como: "Visions d'Esprits, qu'on appelle Spectres" (p. 1); y define espectro como: "Spectre est une imagination d'une substance sans corps, qui se presente sensiblement aux hommes contra l'ordre de nature» (p. 2).

18. En los ejemplos de este segundo capítulo analizado, encontramos la presencia de dos motivos diferentes: por un lado, el tema de la cabeza parlante; por otro, el de la cabeza cortada. El motivo de la cabeza parlante está relacionado con el de los autómatas, seres que hablan y que aconsejan o 
un magistrado de Etolia, que después de su muerte regresará para matar a su hijo nacido al que considerará un monstruo. Nos encontramos ante un relato fantástico en donde Polycrite, el magistrado de la historia, "prit son enfant, le mit en pieces \& le dévora, ne laissant que la tête» (Calmet, 1751: 152 [tome II]), cabeza que comenzará a hablar y a predecir las desgracias que sucederán a su patria.

El segundo relato es el de un soldado muerto en batalla. Después de una batalla entre el rey Antíochus y los romanos, un soldado llamado Buptages resultó muerto «demeuré mort sur le champ de bataille, blessé de douze plaies mortelles, se leva tout d'un coup, \& commença à menacer les Romains des maux qui leur devoient arriver de la part des peuples étrangers, qui devoient détruire l'Empire Romain» (Calmet, 1751: 152 [tome II]), devorado por lobos, solamente quedó la cabeza del soldado, que conjuró múltiples amenazas contra los romanos.

Por último, referiremos la historia de Gabinius, soldado de Augusto al cual "la tête coupée par ordre du jeune Pompée» (Calmet, 1751: 153 [tome II]), pero de tal manera que la cabeza quedó prendida al cuello como si de un fino hilo se tratara. Gabinius afirmó que volvía del infierno para predecirle a Pompeyo cosas muy importantes. En estos tres relatos, podemos apreciar la presencia de cabezas parlantes, tomadas por fantasmas que, conjurados por un demonio, vuelven a la vida para amenazar y buscar venganza.

El motivo de la decapitación está presente en multitud de culturas. En el folclore y en las leyendas, la identificación de espíritus malignos, poseídos por el diablo, en forma de cabezas que hablan que recoge Calmet en sus escritos, aparece como espíritus vengativos. En la cultura malaya, se habla de "cabezas voladoras que llevan al cuello un "collar" [...] hecho con los intestinos de animales" (Santamaría, 2009: 146), una especie de chupasangre llamados Pennangalan. También encontramos los Layak de Bali, seres con cabeza voladora con colmillos e intestinos colgando (Hall, 2011: 135). Pero será a partir de la cultura celta y alemana

predicen el futuro. Pueden ser seres sobrenaturales que se manifiestan a través de sus iconos o representaciones materiales. El tema del autómata parte del motivo siniestro freudiano de la mutilación de las partes separadas del cuerpo. Como tema literario aparece en nuestra literatura en el Quijote, concretamente en la segunda parte del libro, en el capítulo LXII Que trata de la aventura de la cabeza encantada, con otras niñerías que no pueden dejar de contarse, donde don Quijote cree a la cabeza parlante, prodigio según él de la brujería, pero que en realidad es un truco de feria. Es curioso también constatar, tal y como afirma RoAs (2006: 51-52) que «lo maravilloso encontró vías de expresión en los diversos espectáculos populares que recorrieron las ciudades españolas durante el siglo XVIII. [...] Los autómatas fueron también una fuente de placer maravilloso por ese juego con los límites entre lo mecánico y lo humano. [...] Motivo recurrente de la posterior narrativa fantástica romántica».

Respecto al segundo motivo, el de la cabeza cortada, podemos afirmar que sus fuentes artísticas y literarias provienen de la antigüedad, autores como Augusto, Diodoro de Sicilia o Estrabón hablarán en sus escritos de la costumbre de cortar las cabezas de los enemigos después de las batallas. BlázQuez (1978), en su estudio Cabezas cortadas, llega a la conclusión de la gran proliferación de cabezas cortadas en el arte celta de la península ibérica. Elementos que pasarán, posteriormente, al arte decorativo de la época medieval, convirtiéndose más tarde en tópico literario y motivo de la literatura decimonónica. 
cuando el motivo entre a formar parte de numerosas leyendas que influenciarán a los primeros romances literarios como el de Sir Gawain and the Green Knight (Sir Gawain y el Caballero verde) de finales del siglo XIV, donde el tópico de la decapitación que formaba parte de la mitología celta pase a literaturizarse. Pero si los relatos de cabezas de fantasmas llegaron a popularizarse literariamente fue gracias al cuento de Washington Irving Sleepy Hollow (El jinete sin cabeza) (1820); un cuento de terror, en el que el espectro del Jinete es el fantasma de un antiguo soldado bessiano alcanzado por una bala de cañón en la cabeza.

El siguiente capítulo es el XXXVIII y lleva por título: Exemples des personnes qui se sont promis de se donner après leur mort des nouvelles de l'autre monde. En este capítulo, Calmet narrará seis historias de aparecidos, que en vida se prometieron que, cuando muriesen, volverían para dar noticias del Más Allá a sus allegados.

La primera narración nos cuenta la historia de dos amigos: "L'histoire du Marquis de Rambouillet, qui apparut après sa mort au Marquis de Précy» (Calmet, 1751: 170 [tome II]). Estos dos amigos prometieron que el primero de los dos que muriese volvería para dar referencias al otro de todo lo que había visto en el Más Allá. Rambouillet partió a la guerra en Flandes y Précy quedó, muy enfermo, en París. Transcurrido un tiempo de la partida de Rambouillet, y encontrándose en cama todavía su amigo, este sintió una presencia en su habitación y descorriendo las cortinas de su cama, vio allí de pie a Rambouillet. Contento por la presencia de su amigo, intentó abrazarlo, pero aquel, separándose de él, habló, diciéndole que todo lo que ellos pensaban de la otra vida era cierto, y que aún estaba a tiempo de enmendar sus faltas, ya que, en breve, él también moriría. Cuando Précy, sin prestarle atención, intentó abrazarlo por su inmensa alegría, "mais il n'embrassa que du vent» (Calmet, 1751: 171 [tome II]) y Rambouillet, viendo que su amigo no creía aquello que él le decía, le mostró el lugar de su herida mortal, herida de la que todavía brotaba sangre. Al poco tiempo, el marqués recibió una carta comunicándole que Rambouillet había muerto en batalla; y la predicción que su amigo le había hecho se cumplió, pues el marqués de Pércy murió combatiendo en otra batalla.

El segundo relato, lo refiere Calmet tomado de Pierre le Vénérable, abad de Cluny (1092-1156), que, en su obra De miraculis, narrará la siguiente historia: Godofredo d'Iden fue muerto en combate, en una batalla cuya guerra había sido promovida por un gentilhombre llamado Humberto. A los dos meses de su defunción, Godofredo se apareció a un tal Milon d'Ansa, diciéndole que dijera al tal Humberto que él había perdido su vida en una guerra injusta. Que había muerto sin expiar sus pecados, por lo que le rogaba que Humberto ofreciese misas, limosnas y oraciones por su redención, para, así, poder expiar sus pecados. Y el aparecido sentenció: "Dites-lui que s'il ne vous écoute pas, je ferai contraint d'aller moi-même lui annoncer ce que je viens de vous dire» (Calmet, 1751: 173 [tome II]). Humberto hizo caso omiso a la advertencia, pero evitaba durante la noche estar solo, por miedo a que el espíritu pudiera aparecérsele. Una mañana 
que descansaba en su aposento, vio a Godofredo con traje de batalla mostrándole la herida que lo había matado, herida que se apreciaba como acabada de hacer; le reprochó su conducta y la poca piedad que había demostrado, así como que cesara en su empeño de guerrear diciéndole:

Prenez garde, ajoûta-t-il, que Die une vous traite dans sa rigueur, \& ne vous refuse la miséricorde que vous nous refusez; \& fur-tout gardez-vous bien d'exécuter la résolution que vous avez prise d'aller à la guerre avec le Comte Amedée: si vous y allez, vous y perdrez la vie \& les biens (Calmet, 1751: 173 [tome II]).

Humberto haciendo, por fin, caso de lo que le decía el espectro, peregrinó hasta Jerusalén para expiar sus culpas y redimir a los espíritus, para conseguir su descanso en el Más Allá.

La tercera historia la recoge Calmet del cardenal Baronius ${ }^{19}$, que cuenta la historia de dos filósofos. Michel Mercati ${ }^{20}$ y Marsile Ficin ${ }^{21}$ eran dos amigos filósofos que discutían sobre la inmortalidad del alma y su existencia después de la muerte. Ambos amigos prometieron que el primero de ellos que muriese volvería para dar noticias al otro de la otra vida. Una mañana que Michel estaba estudiando en su despacho, oyó cascos de caballos que se acercaban a su ventana y una voz que decía: "Michel, Michel, rien n'est plus vrai que ce qu'on dit de l'autre vie» (Calmet, 1751: 175 [tome II]). Asomado a la ventana vio a Marsile galopando a toda velocidad. Pero Marsile vivía en Florencia y se apareció a su amigo a la misma hora en que había muerto. A partir de este momento, Michel vivió según las normas del cristianismo de una manera ejemplar.

El siguiente relato viene sacado por Calmet de la vida del beato Joseph de Lionisse, capuchino misionero, y que dice así: dos monjes, uno de ellos Joseph, se prometieron un día que conversaban de los preceptos que hay que cumplir para servir a Dios, y sus leyes; que, si uno de los dos moría primero, regresaría para contar al otro la recompensa disfrutada en la otra vida, como consecuencia de haber cumplido con todos los deberes del buen cristiano. Y fray Joseph dijo: "Je veux que nous nous promettions mutuellement que celui de nous qui mourra le premier, apparoisse à l'autre, si Dieu le permet ainsi, pour l'instruire de ce qui se passe en l'autre, \& de l'état où il se trouvera" (Calmet, 1751: 176 [tome II]). Al poco tiempo, el compañero de fray Joseph murió de enfermedad, y un día que este se encontraba en su celda, vio que entraba un joven capuchino con rostro completamente aterrado, y Joseph comprendió que era el espectro, el cual le dijo: "Je viens ici comme Dieu l'a permis, pour m'acquitter de ma promesse, \& pour vous dire que j'ai le conheur d'être du nombre des élus par la miséricorde du Seigneur» (Calmet, 1751: 177 [tome II]). Que poder gozar de la misericordia de los

19. Caesar Baronius (1538-1607).

20. Michele Mercati (1541-1593), científico, médico y filósofo.

21. Marsilio Ficino (1433-1499), humanista italiano, médico y filósofo. 
elegidos era muy difícil, y que Dios sopesaba con mucha rectitud los actos malos y los buenos actos, pero que si su vida era pura y bondadosa, el Dios indulgente lo dejaría encontrar la felicidad de los elegidos. Y una vez dicho todo esto, "le Fantôme disparut» (Calmet, 1751: 177 [tome II]).

La penúltima historia de este capítulo versa sobre el arzobispo Gamrate, al cual se le aparecerá un antiguo amigo de fechorías, y dice así: Pierre Gamrate, obispo de Cracovia, fue trasladado al arzobispado de Gnesne. Dicho prelado había disfrutado, en su juventud, de una vida licenciosa y de fechorías, por lo cual, ahora en su vejez y con su cargo, se dedicaba a socorrer a los pobres otorgándoles comida y ropajes. Un día, la víspera de una gran fiesta eclesiástica, se le apareció un antiguo compañero de fechorías de su juventud, Curosius, que hacía ya tiempo que había fallecido, para advertirlo de lo que le había pasado. Curosius había matado a un hombre por haber blasfemado contra la Virgen María, y a la hora de su muerte, la misma Virgen intercedió ante Dios para que le perdonara sus pecados. Y así le dijo a Gamrate:

Pour vous, vous n'avez plus que six mois à vivre; \& je suis envoyé pour vous avertir, que Dieu en considération de vos aumônes, \& de votre charité envers les pauvres, veut vous faire miséricorde, \& vous attend à pénitence. Profitez du tems, \& expiez vos péchés passés (Calmet, 1751: 179 [tome II]).

Después de lo cual el fantasma desapareció. El arzobispo, turbado por la presencia y por las palabras, llevó una vida recta y cristiana hasta el fin de sus días.

La última historia de este capítulo relata el caso de la hija de un famoso jurisconsulto llamado Dumoulin ${ }^{22}$. Habiendo sido asesinada en su casa junto a sus hijos, se le apareció durante la noche a su marido, dándole los nombres y apellidos de sus asesinos, clamando así venganza.

El último capítulo analizado será el XXXIX: Extrait des Ouvrages politiques de M. l'Abbé de Saint Pierre, tome 4, pag. 57. Como podemos apreciar, en este capítulo, Calmet se basará en el relato del abad de Saint Pierre en Valogne, el cual, a su vez, contará una historia de un sacerdote de su parroquia al que llamaban Bezuel. El abad oyó el relato del mismo Bezuel durante una cena acontecida el día 7 de enero de 1708. Y la historia dice así: Siendo joven Bezuel estudió con un compañero llamado Desfontaines, cuya amistad fue muy fuerte. El año 1696, siendo los dos jóvenes capuchinos, cuenta Bezuel que su amigo le relató la historia de otros dos amigos que habían prometido darse noticias después de la muerte. El relato de esta historia por parte del propio Bezuel dice: «La histoire de deux amis, qui étoient promis, que celui qui mourroit le premier, viendroit dire de ses

22. Consideramos interesante para la investigación reproducir el texto de Le Loyer que copia Calmet: «La fille de du Molin meurtrie \& homicidee s'estoit mostree de nuit au baillif de Colommiers son maty, veillant \& non dormant, \& lui auroit revelé ses meurtriers \& de ses petits enfans" (Le Loyer. Livre Sixiesme. Chapitre Premier, XV, p. 677. Qu'és Cours de Parlement la questions auroit esté meuë quelquesfois si les Esprits retournoient). 
nouvelles au vivant, que le mort revint, \& lui dit des choses tres surprenantes, sur cela» (Bezüel, 1708: 2). Desfontaines propuso hacer el mismo trato a Bezuel, pero este se negó. Tiempo después, fue tal la insistencia, que al final Bezuel cedió e hicieron un pacto de sangre. Desfontaines partió con su hermano, pero antes de irse me dijo: "Je vous ay promis, [...] que si je mourois avant vous, je viendrois vous le dire, je suis mort» (Bezüel, 1708: 7).

Concretamente el día 31 de julio de 1697, Bezuel se encontró indispuesto, al igual que el día siguiente, lo cual le alteró mucho, pues pensó en alguna enfermedad; y lo mismo le ocurrió al siguiente día. Desvanecido y perdido el conocimiento, fue ayudado por los lacayos del señor al que servía en las faenas de la cosecha, y avanzando por una escalera, vio de repente la figura de su amigo Desfontaines. Alarmado vio que este le hacía signos para que se le acercara, y así le dijo: «je suis mort, je me neyai avant hier à la riviere de Caën» (Bezüel, 1708: 7). Ante mi sorpresa, intenté abrazarlo varias veces pero: «je m'aprochay plusieurs fois pour l'embrasser; mais il me parut que je n'embrassois rien» (Bezüel, 1708: 8). El amigo retornaba de la muerte para encomendarle un encargo, para decirle que avisara a su hermano y a sus padres de su fallecimiento:

Il me pria quand son frere seroit revenu, de lui dire certaines choses pour dire à son père, \& à sa mere, il me pria de dire sept Pseaumes qu'il avoit eus en penitence le Dimanche précedent, \& qu'il n'avoit pas encore recitez; ensuite il me recommanda encore de parler à son frere (Bezüel, 1708: 9-10).

El fantasma desapareció después de encomendarle estas palabras. Pero como pasó más de un mes, y Bezuel no llevó a cabo el encargo de su amigo, este se le apareció dos veces más de noche, para reprocharle que no hubiera cumplido con su promesa. Cuando, por fin, Bezuel habló con el hermano de Desfontaines, el fantasma nunca más se le volvió a aparecer.

Calmet, en esta historia, sin pretender juzgar al venerable abad de Saint Pierre, continúa reafirmando sus tesis: si las almas pueden volver de la muerte, solamente será porque Dios ha permitido a estas almas separadas del cuerpo aparecerse a los vivos. Habla de apariciones sobrenaturales, por tanto, de milagros, y nos dice:

Le retour des Ames \& leur apparition n'est pas une chose naturelle, ni qui soit du choix des Trépassés. C'est un effet surnaturel \& qui tient du miracle [...].

Le Retour des Ames, leurs apparitions, l'exécution des promesses que quelques personnes se sont faites de venir dire à leurs amis des nouvelles de ce qui se passe en l'autre monde, n'est pas en leur pouvoir. Tout cela est entre les mains de Dieu (Calmet, 1751: 192-193 [tome II]).

Respecto a los tópicos que Calmet recoge en su tratado, es evidente que el motivo de la vuelta del Más Allá entre amigos o conocidos predomina en todas estas historias de estos dos últimos capítulos. La vuelta del espectro, sobre todo, 
para dar referencias de la existencia de la otra vida de la creencia cristiana, y base fundamental de esta teología, posibilita que todas las historias de este capítulo estén relacionadas con este motivo. El espíritu advierte y aconseja enmendar aquellos comportamientos que no hagan llevar una vida cristiana a los aparecidos, para expiar sus pecados y poder rectificar sus actos, y, así, alcanzar la salvación y la vida eterna. Conceptos, todos ellos, pertenecientes a la teología y a las creencias de la época y de nuestro autor.

Las historias literarias de fantasmas ${ }^{23}$ no suelen basarse en estas premisas de augurios del Más Allá, pero sí que coinciden, frecuentemente, con el motivo de la historia contada por el abad, la joven asesinada que clama venganza. Este tópico, la venganza, es uno de los motivos más comunes en las historias de fantasmas, y ya desde los primeros relatos se encuentra presente en la base de la literatura. Uno de los primeros autores en contribuir en la creación del tópico literario del fantasma será Shakespeare, ya que tal y como afirma Chaplin (2011: 11) «The notion of a uniquely English literary tradition was beginning to take shape in this century and central to its formation was the figure of William Shakespeare, whose plays the tragedies especially, had a marked influence on gothic fiction».

En 1764, con la publicación de The Castler of Otranto ${ }^{24}$ (El castillo de Otranto) de Horace Walpole, se inicia uno de los relatos que dará paso al movimiento conocido como "Literatura Gótica» ${ }^{25}$. El esquema narratológico de la historia es el clásico de la época: la usurpación del trono, la aparición de un espectro, la venganza y la restitución de la usurpación. Pero la repercusión de esta obra, dentro de la narrativa de fantasmas, podemos afirmar que fue decisiva, así como la descripción del espectro del castillo: «La figura se dio la vuelta y Frederic vio las mandíbulas descarnadas y las cuencas vacías de un esqueleto, envuelto en la capucha de un ermitaño» (Walpole, 2008: 179).

23. Otras historias de fantasmas de la era victoriana y donde los motivos del regreso varían según el relato son: Rodrigue, ou la Tour enchantée (Rodrigo o la torre encantada) (1800) de Françoise de Sade; The Botathen Ghost (El fantasma de Botathen) (1867) de Robert Stephen Hawker; Madam Crowl's Ghost (El fantasma de Madam Crowl) (1871) de Joseph Sheridan Le Fanu; The Open Door (La puerta abierta) (1882) de Margaret Oliphant; The Canterville Ghost (El fantasma de Canterville) (1887) de Oscar Wilde; John Charrington's Wedding (La boda de Jobn Charrington) (1891) de Edith Nesbit. Para más información sobre el motivo del fantasma (Ghost Story), consultar: HuRwOOD (1974), BRIGGS (1977), COX y Gilbert (1989).

24. Si es interesante esta obra, además de por ser de las primeras del género, es porque tiene relación con algunas de las historias de Calmet ya que, evocations of the supernatural are vivid and extravagant, much more so, in fact, than in certain later fictions which tended to minimise or explain away supernatural interventions" (CHAPLin, 2011: 46).

25. El motivo del fantasma será uno de los principales tópicos de los cuentos de este tipo de literatura porque, tal y como afirma THURSTON (2012: 7), "The ghost is thus properly unspeakable, anamorphic ally skewed away from the familiar fictional discursive mesh of narrative reality: and it is this non-discursivity that will emerge as the primary source [...] of the incessant power and undying fascination of the ghost story». 
La venganza está en la base de muchas narraciones de esta clase de literatura, porque da pie a la trama del relato y presagia su final. En 1822, Nodier escribe dentro de su obra Infernaliana, un "Conte Noir" titulado Spectre qui demane vengeance (El espectro que pide venganza) contextualizado en el siglo XIII, en el que el padre de la bella Abélina es asesinado por el conde de Belmonte, señor del lugar. "Le fantôme» se aparece a Abélina y le dice que "le tyran de Belmonte m'assassiner» (Nodier, 1822: 114) y pide "songes à me venger» (Nodier, 1822: 115). Al final, se profetiza la caída del tirano y esta se cumple.

La venganza es también el motivo del relato Fisher's Ghost (El fantasma de Fisher) (1859) de John Lang, donde la aparición del fantasma del protagonista deja al descubierto el asesinato de este, por parte de su vecino, que anhelaba sus tierras. El espectro aparece para señalar el lugar en el que se encuentra el cadáver, para que pueda ser sepultado y también, para señalar a su asesino. Por último, comentaremos el relato de Wilkie Collins Miss Jéromette and the Clergyman (Mis Jéromette y el clérigo) (1875), en el que se repetirá el tópico de la joven asesinada que se aparece en forma de fantasma para delatar a su asesino, fantasma que aparecerá envuelto, siempre, en una espesa niebla.

\section{CONCLUSIONeS}

Hemos constatado en esta investigación que la tratadística dieciochesca contribuyó, de manera decisiva, en la creación de tópicos que pasarían, posteriormente, a la literatura, en este caso analizando relatos del benedictino Calmet y tomando como referencia sus historias de aparecidos. La creencia en los espíritus que retornan del Más Allá en forma de espectros, de fantasmas que molestan a los vivos, está presente en todas las culturas por el miedo que representa la muerte en sí misma, y en concreto en el tópico del fantasma, por la creencia que la "mala muerte» tenía sobre el no descanso del alma del difunto. La fusión del folclore y las creencias cristianas influirán mucho más en estos conceptos, ya que el terror que simboliza en el subconsciente colectivo el convencimiento de la creencia en un Más Allá, que a la vez conlleva un posible retorno del muerto, por causas de una mala praxis a la hora de ser enterrado, ha atemorizado, constantemente, al hombre. No respetar estas ceremonias comportaba la transformación de los muertos en revenants y fantasmas. Los ritos funerarios siempre han estado presididos por hechos y acciones que pretendían contentar a los muertos y procurar su reposo final, y esta creencia es universal.

Pero la irrupción del cristianismo produjo un cambio radical en los ritos funerarios, y la convicción de la resurrección del alma dio paso a las historias de espectros y fantasmas. La tratadística dieciochesca pretendía aunar la mayoría de saberes de la época, y uno de sus principales referentes será el padre Calmet. Sus escritos e historias de muertos vueltos de la tumba, que retornaban, evidentemente, por motivos piadosos o vengativos servirán, en muchos casos, para 
contribuir a la ferviente imaginación de muchos de los escritores analizados, así como a muchas de las historias de fantasmas ${ }^{26}$ que la literatura popularizará a partir, sobre todo, del siglo XIX.

Evidentemente, la temática cristiana del retorno del Más Allá para obtener el perdón se transformará, se adaptará o se cambiará respecto a los intereses literarios de los escritores. Pero, en realidad, la base antropológica de estos relatos procede de las leyendas y el folclore, recogido y recopilado por autores como Calmet. Se crearán los tópicos, a partir de las historias narradas en este tratado y en otros, que configurarán los elementos básicos de la narrativa literaria de fantasmas: el espíritu que vuelve para pedir (misas, el perdón, cumplir una promesa, peregrinar, vengarse o predecir el futuro...). La naturaleza de estos fantasmas, que en los relatos literarios también serán benévolos o malévolos, surge de la fascinación que sobre los humanos ejerce el concepto de la muerte y la esperanza de la existencia de otra vida. Los patrones narrativos que hemos estudiado convergerán y serán similares en las historias del folclore y en la literatura.

Las visiones de los aparecidos siempre se producirán entre conocidos (amigos, esposa, hijo...). En un primer momento, la aparición irá acompañada de incredulidad y miedo, miedo que en los cuentos literarios puede llegar a convertirse en terror. El fantasma siempre se aparece en soledad, nunca a grupos de gente. A veces, da muestras de arrepentimiento por sus malas acciones; a veces, también aprovecha la noche, la oscuridad (anticipando una de las características fundamentales del relato gótico), incluso el descanso del protagonista para aparecérsele; lo acompaña la niebla (elemento gótico), que será un recurso básico de

26. Respecto a la literatura nacional, la española, podemos señalar que los relatos sobre apariciones de muertos que vuelven para vengarse o para poder conseguir el descanso eterno son un recurso presente en la literatura decimonónica. Los cuentos sobre fantasmas aparecerán sobre todo publicados en la prensa del XIX (La Crónica, El Entreacto, La Esperanza, La Ilustración...), ya que el público de esta época gustaba de relatos de terror sobrenatural y de sucesos extraños y sobrenaturales, asentándose así el germen de la literatura fantástica. Relatos basados en apariciones humanas como: $E l$ Vivac (s. n. 1845), Las Willis (s. n. 1845), La dama blanca de Baden (s. n. 1853) o La fuente del Montal (Augusta Ferrán, 1866) (Trancón, 2000).

Pero tampoco hemos de olvidar que la novela gótica empezó a cultivarse tarde en nuestro territorio. LÓPEZ SANTOS (2010) habla de novela gótica racional terrorífica que busca el miedo escondido tras los pliegues de la veracidad histórica y la novela gótica irracionalista, que abandona el componente sobrenatural y se recrea en el placer del horror, para dar rienda suelta a la monstruosidad y que juega con la angustia y el sufrimiento a través de una lección moral bastante debilitada. A la novela gótica de impulso racional pertenecen dos relatos de fantasmas, La torre gótica o El Espectro de Limberg (1831), atribuida a Pascual Pérez Rodríguez. Texto en el que aparece un fantasma que será responsable de los sucesos extraños que acontecerán en el castillo, «apariciones de fantasmas que se asociarán a la justicia divina” (López SANTOS, 2010: 217), y que, hasta que no se haga justicia por una muerte, no habrá perdón y descanso eterno. Y un segundo relato, La urna sangrienta (1834), del mismo autor, donde «el fantasma [...] se manifiesta en toda su complejidad y valiéndose de todo el artificio de terror» (López SANTOS, 2010: 218), fantasmas de buenas intenciones que advierten de lo que puede ocurrir para disuadir de los actos malvados. 
estas narraciones literarias. El fantasma siempre tiene una misión que cumplir, y cuando esta se haya cumplido, el espíritu desaparecerá, propiciándose así el descanso eterno; y cuando el fantasma clama venganza, o restitución de su muerte, y es enterrado debidamente, también desaparecerá. Hemos pretendido demostrar, en este artículo, la relación tan directa que existe entre las historias que Calmet recopiló en su tratado y cualquier cuento de fantasmas de la literatura gótica de los que hemos nombrado, ya que tan fantásticos nos parecen unos relatos, como los otros; porque tal y como afirma Roas (2011: 92): "lo fantástico -a través de vampiros, fantasmas, dobles y el resto de figuras y motivos del imaginario sobrenaturaltiene que ver con los miedos colectivos [...] con todo aquello que escapa a los límites de la razón». Por tanto, también podemos afirmar que será en pleno siglo XVIII cuando la literatura fantástica iniciará su andadura, pues entre las historias de Calmet, que hemos analizado, encontraremos sucesos extraordinarios y sobrenaturales, que producen terror, y que posteriormente, cuando aparezca la literatura fantástica, pasarán a ser motivos que provocarán el mismo desasosiego e intranquilidad entre el público que los relatos de nuestro autor provocaron en su época.

\section{BiBLIOGRAFÍA}

AguirRe CASTRO, Mercedes. "Caracterización y representación de los fantasmas en la antigua Grecia». En Aguirre Castro, Mercedes; Delgado Linacero, Cristina y GonzÁlezRivas, Ana (eds.). Fantasmas, aparecidos y muertos sin descanso. Madrid: Abada Ediciones, 2014.

Agustí APARISI, Carme. "Calmet y el vampiro: un personaje del mal. Aproximación desde la antropología a la literaturización del fenómeno vampírico». Cuadernos de Ilustración $y$ Romanticismo. Revista digital del Grupo de estudios del siglo XVIII. Universidad de Cádiz, 2016, 22, 179-203.

Bezuel, M. Histoire d'une Aparition. Gallica. Bibliothèque Nationale de France, 1708 [consulta en línea: http://gallica.bnf.fr/ark:/12148/bpt6k6471480t/f6.item.r=Bezuel.zoom; 25/05/2017].

Blázquez MarTínez, José María. "Cabezas cortadas". Historia 16, 1978, núm. 26, pp. 33-39. Edición digital de la Biblioteca Virtual Miguel de Cervantes [2000].

Buxton, Richard. "Fantasmas y religión entre los griegos: contextos y control». En AGuirRe Castro, Mercedes; Delgado Linacero, Cristina y González-Rivas, Ana (eds.). Fantasmas, aparecidos y muertos sin descanso. Madrid: Abada Ediciones, 2014.

CALMET, Dom Augustin. Traité sur les Apparitions des Esprits, et sur les vampires, ou les Revenans de Hongrie, de Moravie, Ec, tome I. Paris: Debure l'aîné. Gallica. Bibliothèque Nationale de France, 1751 [consulta en línea: http://gallica.bnf.fr/ark:/12148/ bpt6k68179p?rk=21459;2; 15/01/2015].

CALMET, Dom Augustin. Traité sur les Apparitions des Esprits, et sur les vampires, ou les Revenans de Hongrie, de Moravie, Ec, tome II. Paris: Debure l'aîné. Gallica. Bibliothèque Nationale de France, 1751 [consulta en línea: http://gallica.bnf.fr/ark:/12148/ bpt6k68180w?rk=42918;4; 15/01/2015]. 
Calmet, Dom Augustin. Tratado sobre los Vampiros. Traducción de Lorenzo Martín del Burgo. Prólogo de Luis Alberto de Cuenca. Unión Europea. Madrid: Reino de Cordelia ([1751] 2009).

Collin De Plancy, Jacques Auguste Simon. Histoire des Vampires et des Spectres Malfaisans. París: Dupont P. Gallica. Bibliothèque nationale de France, 1820 [consulta en línea: http://tolosana.univ-toulouse.fr/notice/134137337; 7/12/2016].

Collin De Plancy, Jacques Auguste Simon. Dictionnaire infernal. Paris: Henri Plon. Gallica. Bibliothèque Nationale de France, 1863 [consulta en línea: http://gallica.bnf.fr/ ark:/12148/bpt6k5754923d/f687; 18/03/2015].

Chaplin, Sue. Gothic Literature. London: York Press, 2011.

Delgado Linacero, Cristina. «Seres maléficos, fantasmas y espíritus en Mesopotamia». En Aguirre Castro, Mercedes; Delgado linacero, Cristina y González-Rivas, Ana (eds.). Fantasmas, aparecidos y muertos sin descanso. Madrid: Abada Ediciones, 2014.

EZAma GIL, Ángeles. «El fantasma en algunos cuentos españoles del siglo XX». En PONT, Jaume (ed.) Brujas, demonios y fantasmas. Lleida: Edicions de la Universitat de Lleida, 1999.

GonZÁlez SAlVAdor, Ana. "Continuidad del fantasma. (Un ejemplo: Las hortensias de Felisberto Hérnández)». En PONT, Jaume (ed.). Brujas, demonios y fantasmas. Lleida: Edicions de la Universitat de Lleida, 1999.

GONZÁLEZ-RIVAS FERNÁNDEZ, Ana. «Plinio el Joven y la imagen del fantasma en la ilustración literaria del siglo XIX». En Aguirre Castro, Mercedes; Delgado Linacero, Cristina y GONZÁlEZ-RIVAS, Ana (eds.). Fantasmas, aparecidos y muertos sin descanso. Madrid: Abada Ediciones, 2014.

HaLl, Derek. Mitos y romanticismo de los vampiros. Barcelona: Obelisco, 2011.

Lecouteux, Claude. Histoire des Vampires. Autopsie d'un mythe. Paris: Imago, 2009a.

LeCouteux, Claude. Fantômes et Revenants au Moyen Âge. Paris: Imago, 2009b.

LE LOYER, Pierre. Discours et histoire des spectres, visions et apparitions des esprits, anges, demons, et ames, se monstrans visibles aux hommes. Paris: Chez Nicolas Byon. Gallica, 1605. Bibliothèque nationale de France [consulta en línea: http://gallica.bnf.fr/ ark:/12148/bpt6k5545032t; 26/04/2017].

López SANTOS, Miriam. La novela gótica en España (1788-1833). Vigo: Academia del Hispanismo, 2010.

MARIGNY, Jean. Le réveil des vampires. Paris: Gallimard, 1993.

MCCORristine, Shane. Spectres of the Self: Thinking about Ghosts and Ghost-Seeing in England, 1750-1920. Cambridge: Cambridge University Press, 2010.

MORILLAS VeNTURA, Enriqueta. "Los fantasmas rioplatenses de fines del siglo XIX». En PONT, Jaume (ed.). Brujas, demonios y fantasmas. Lleida: Edicions de la Universitat de Lleida, 1999.

Ogden, Daniel. "Fantasmas romanos». En Aguirre Castro, Mercedes; Delgado Linacero, Cristina y GONZÁlez-Rivas, Ana (eds.). Fantasmas, aparecidos y muertos sin descanso. Madrid: Abada Ediciones, 2014.

Réalité de la Magie, et des Apparitions, ou Contrepoison du Dictionnaire Infernal (1819), Paris: Brajeux. Books.google.es.

ROAS, David. "Voces del otro lado: el fantasma en la narrativa fantástica». En PONT, Jaume (ed.). Brujas, demonios y fantasmas. Lleida: Edicions de la Universitat de Lleida, 1999.

ROAS, David. De la maravilla al horror. Pontevedra: Mirabel Editorial, 2006.

ROAS, David. Tras los limites de lo real. Madrid: Ed. Páginas de Espuma, S.L., 2011.

Santamaría, Simonetta. Vampiros desde Drácula hasta Twilight. Madrid: Paraninfo, 2009. 
Thompson, Stith. Motif-index of folk-literature: a classification of narrative elements in folktales, ballads, myths, fables, medieval romances, exempla, fabliaux, jest-books, and local legends. Revised and enlarged. Edition Bloomington, Indiana: University Press, 1955-1958 [consulta en línea: http://www.ruthenia.ru/folklore/thompson/; 20/01/2016a.

TRANCÓN LAgUnAS, Montserrat. La literatura fantástica en la prensa del Romanticismo. Institució Alfons El Magnànim. Diputació de València, 2000.

THURSTON, Luke. Literary Ghost from the Victorians to Modernism. New York: Routledge, 2012.

Fuentes literarias consultadas

BRADDON, Mary Elizabeth. "En la abadía de Chrighton». En Cuentos de fantasmas de la era victoriana. Murcia: Tres Fronteras Ediciones, 2011.

ColLins, Wilkie. "La señorita Jéromette y el pastor». En Cuentos de fantasmas de la era victorian. Murcia: Tres Fronteras Ediciones, 2011.

DANTE, Alighieri. Divina Comedia. Edición de Ángel Chiclana. Madrid: Austral poesía, 2010.

Defoe, Daniel. "La aparición de Mrs. Veal». En Antología de cuentos de terror. 1. Selección de Rafael Llopis. Madrid: Alianza Editorial, 2015.

GaIL, Teresa; Garrido, Manuel et al. Historias de fantasmas y misterio de la antigüedad (Antologia). Valencia: Editilde S.L., 2010.

LANG, John. "El fantasma de Fisher». En Cuentos de fantasmas de la era victoriana. Murcia: Tres Fronteras Ediciones, 2011.

NODIER, Charles. Infernaliana, ou anecdotes, petits romans, nouvelles et contes. Paris: Sanson. Gallica, 1822. Bibliothèque Nationale de France [consulta en línea: http://catalogue.bnf.fr/ark:/12148/cb31021161n; 25/05/2017].

ScotT, Walter. "Relato de Willie el Vagabundo». En Antología de cuentos de terror. 1. Selección de Rafael Llopis. Madrid: Alianza Editorial, 2015.

WalPole, Horace. El Castillo de Otranto. Prólogo, traducción y notas de María Engracia Pujals. Madrid: Alianza editorial. Biblioteca de fantasía y terror, 2008. 